\title{
Antibacterial and Anti-Virulence Effects of Furazolidone on Trueperella Pyogenes and Pseudomonas Aeruginosa
}

\section{Qin Chen}

Sichuan University

\section{Kelei Zhao}

Sichuan Industrial Institute of Antibiotics, Chengdu University

Heyue Li

Sichuan University

Kanghua Liu

Sichuan University

\section{Jing Li}

Sichuan Industrial Institute of Antibiotics, Chengdu University

\section{Yiwen Chu}

Sichuan Industrial Institute of Antibiotics, Chengdu University

\section{Balakrishnan Prithiviraj}

Dalhousie University

\section{Bisong Yue}

Sichuan University

Xiuyue Zhang ( $\square$ zhangxiuyue@scu.edu.cn )

Sichuan University

\section{Research Article}

Keywords: Forest musk deer, Trueperella pyogenes, Pseudomonas aeruginosa, quorum-sensing, furazolidone, anti-virulence

Posted Date: August 10th, 2021

DOl: https://doi.org/10.21203/rs.3.rs-778315/v1

License: (c) (i) This work is licensed under a Creative Commons Attribution 4.0 International License. Read Full License 
Version of Record: A version of this preprint was published at BMC Veterinary Research on March 24th, 2022. See the published version at https://doi.org/10.1186/s12917-022-03216-5. 


\section{Abstract}

Background: Trueperella pyogenes and Pseudomonas aeruginosa are two important bacterial pathogens closely relating to the occurrence and development of forest musk deer respiratory purulent disease. Although $T$. pyogenes is the causative agent of the disease, the subsequently invaded $P$. aeruginosa will predominate the infection by producing a substantial amount of quorum-sensing (QS)-controlled virulence factors, and co-infection of them usually creates serious difficulties for veterinary treatment. In order to find a potential drug that targets both $T$. pyogenes and $P$. aeruginosa, the antibacterial and antivirulence capacities of 55 compounds, which have similar core structure to the signal molecules of $P$. aeruginosa QS system, were tested in this study. By performing a series of in vitro screening experiments to assess the effects of these compounds.

Results: We identified that furazolidone could significantly inhibit the growth of mono-cultured $T$. pyogenes or in the co-culture with $P$. aeruginosa. Although the growth of $P$. aeruginosa could also be moderately inhibited by furazolidone, the results of phenotypic identification and transcriptomic analysis further revealed that furazolidone had remarkable inhibitory effect on the biofilm production, motility, and QS system of $P$. aeruginosa. Moreover, furazolidone could efficiently protect Caenorhabditis elegans from $P$. aeruginosa infection under both fast-killing and slow-killing conditions.

Conclusions: This study reports the antibacterial and anti-virulence abilities of furazolidone on $T$. pyogenes and $P$. aeruginosa, and provides a promising strategy and molecular basis for the development of novel anti-infectious drugs to dealing with forest musk deer purulent disease, or other diseases caused by $T$. pyogenes and $P$. aeruginosa co-infection.

\section{Background}

Forest musk deer (Moschus berezovskii) is an important economic animal and is endemic to China. The musk secreted by male forest musk deer is a precious Chinese medicine and important raw material of high-grade spice. Purulent disease usually manifests as purulent lesions on the epidermis, uterus, and internal organs, and is one of the main reasons hindering the growth of forest musk deer population [1, 2]. Compared to the body surface abscesses which can be easily observed and removed artificially, the internal purulent lesions are extremely harmful due to the complex bacterial composition, and also are difficult to be detected in time $[1,3]$. Therefore, internal abscesses are usually found dying deer and have brought huge challenge and economic loss to forest musk deer breeding industry.

Our prior work has shown that Trueperella pyogenes and Pseudomonas aeruginosa are two main bacterial pathogens in the respiratory suppurative lesion of forest musk deer, with $T$. pyogenes as the primary pathogen and $P$. aeruginosa as the dominate species in the lateral stage $[1,2,4]$. T. pyogenes is Gram-positive, pleomorphic, non-spore forming, inactive, non-enveloped and facultative anaerobic belonging to the family Actinomycetaceae, and a resident bacterium of the skin and mucous membranes of the animal respiratory tract, digestive tract, and genitourinary tract $[5,6,7,8]$. This microorganism can reduce the oxygen content and oxidation-reduction potential of the infection site by producing the key 
virulence factor pyolysin through fermentation metabolism, and this process is beneficial to other bacterial species $[9,10]$.

$P$. aeruginosa is a ubiquitous opportunistic Gram-negative bacterium that can infect a variety of host tissues and cause acute and chronic infections $[11,12]$. It is well-recognized that the quorum-sensing (QS) system, which is activated by specific signal molecules (acyl-homoserine lactones, AHLs) and coordinates the production of virulence factors and intracellular metabolism of many bacterial species, plays an important role in the processes of bacterial invasion and cell-cell communications $[3,13,14,15]$. The QS system of $P$. aeruginosa is composed of three hierarchically arranged regulatory networks. The las- and rhl-QS systems have complete signal molecule synthesis proteins (Lasl/Rhll) and regulatory proteins (LasR/RhIR), and the activation of $r h /$ is mainly depended on las. The pqs system only has the regulatory protein $p q s R$, and the activation of which requires Pseudomonas quinolone signal from other pathways co-regulated by lasR and $r h / R[14]$.

It is considered that inhibiting the virulence of pathogenic bacteria by targeting the QS system is a promising strategy for the development of novel anti-infectious drugs, namely QS inhibitors or antivirulence drugs [16]. We have previously shown that the natural AHL signals of $P$. aeruginosa QS system could inhibit the growth and virulence of $T$. pyogenes [17]. P. aeruginosa has strong competitive advantage over T. pyogenes when any of the three QS regulators of $P$. aeruginosa was knocked out [18]. Therefore, we hypothesize that there might be a kind of drug can simultaneously inhibit the growth or virulence of T. pyogenes and P. aeruginosa. Hence, in this study, we tested the antibacterial and anti-QS activities of 55 small molecule drugs with similar core structure to that of $P$. aeruginosa QS signals. Finally, we identified that furazolidone could inhibit the growth of $T$. pyogenes and $P$. aeruginosa and showed a strong inhibitory activity on the QS system of $P$. aeruginosa.

\section{Results}

\section{Screening of compounds inhibit the growth of T. pyogenes}

For the 55 compounds with similar core structure to the AHL signals of $P$. aeruginosa QS system, we first tested the inhibitory effects of them on the growth of T. pyogenes. We found that although the majority of the compounds had no effect on the growth of T. pyogenes TP13 in BHI-FBS broth compared with the control, 9 of them were found to have significant growth inhibition effect and 8 have significant growth enhancement effect (Additional file 1 Supplementary Table S1). Nitrofurantoin, nitrofurazone, ronidazole, and furazolidone, which could significantly inhibit T. pyogenes TP13 in a dose-dependent manner (Fig. 1), were selected to treat co-cultured T. pyogenes and P. aeruginosa.

Furazolidone inhibits the growth of T. pyogenes and P. aeruginosa

T. pyogenes TP13 and $P$. aeruginosa PA01 were well-mixed into different ratios (1:1, 1:9 and 9:1) and cocultured in BHI-FBS agar supplemented with $200 \mu \mathrm{M}$ of nitrofurantoin, ronidazole, furazolidone, and nitrofurazone. In agreement with our prior finding that $P$. aeruginosa had an innate growth advantage in 
the competition with T. pyogenes [17], P. aeruginosa would always be the dominant species under the coculture condition with T. pyogenes, irrespective of their initial ratios (Fig. 2). The addition of the compounds, especially nitrofurazone, significantly suppressed the growth of $T$. pyogenes TP13 but had different effects on the growth of $P$. aeruginosa PAO1 in the co-culture. Notably, among of the 4 tested compounds, only furazolidone could simultaneously inhibit the growth of T. pyogenes TP13 and $P$. aeruginosa PAO1 compared to the control (Fig. 2).

\section{Furazolidone inhibits the QS-related phenotypes of P. aeruginosa}

We found that furazolidone showed a dose-dependent growth inhibition effect on $P$. aeruginosa PA01, but would not totally clean them (Additional file 1 Supplementary Fig. S1). Considering the similar core structure of furazolidone to the AHL signals of $P$. aeruginosa QS system, we then tested the possibility that furazolidone might have a negative effect on the QS regulation of $P$. aeruginosa PAO1. Because the rapid population proliferation of $P$. aeruginosa using adenosine or skim milk as the sole carbon source requires the intracellular hydrolase or extracellular protease elastase that are positively regulated by the QS system, and thus the growth status of $P$. aeruginosa under these conditions can be used to preliminarily evaluate the performance of QS system [19]. As shown in Table 1, furazolidone significantly suppressed the growth of $P$. aeruginosa PAO1 on M9-adenosine plates, and had a dose-dependent inhibition effect on the production of extracellular proteases on M9-skim milk plates. Moreover, we further showed that the production of biofilm and pyocyanin and the swimming and twitching motilities of $P$. aeruginosa PAO1 could also be significantly inhibited by furazolidone (Fig. 3).

Table 1

Inhibitory effect of furazolidone on the growth of $P$. aeruginosa PA01 on M9-adenosine and M9-skim milk plates.

\begin{tabular}{|lll}
\hline Furazolidone $(\mu \mathrm{M})$ & M9-adenosine & M9-skim milk $(\mathbf{c m})^{\mathbf{b}}$ \\
\hline 0 & + & $1.72 \pm 0.061$ \\
\hline 50 & - & $1.57 \pm 0.050 \star \star \star \star$ \\
\hline 100 & - & $1.41 \pm 0.074 \star \star \star \star$ \\
\hline 200 & - & $1.32 \pm 0.050 \star \star \star \star$ \\
\hline a " + ", Normal growth. " $-"$, Inhibited growth. \\
\hline $\begin{array}{l}\text { b Data shown are the mean } \pm \text { standard deviation of nine independent experiments. One-way ANOVA, } \\
\star \star \star \star \star\end{array} p<0.0001$.
\end{tabular}

\section{Furazolidone inhibits the QS-regulation of P. aeruginosa}

To further investigate the effect of furazolidone on the QS regulation of $P$. aeruginosa, RNA-sequencing was then used to profile the global transcription change of furazolidone-treated $P$. aeruginosa PAO1. The results showed that compared to the control, 465 up-regulated genes and 107 down-regulated genes were 
identified in P. aeruginosa PA01 cultured in LB broth supplemented with $200 \mu \mathrm{M}$ of furazolidone (Fig. 4A and Additional file 2). Prediction of KEGG pathway revealed that the functions of flagellar assembly, bacterial chemotaxis, propanoate metabolism, ribosome, and degradation of valine, leucine and isoleucine were significantly enriched among the up-regulated genes $(p<0.05)$, while QS system was the sole significantly enriched KEGG term among the down-regulated genes (Fig. 4B).

We then explored the suppression effect of furazolidone on P. aeruginosa QS system in more detail by applying all the significantly changed genes to the list of QS-induced genes previously released by Schuster [20]. We found that among the 315 QS-induced genes, 63 of them including the typical genes relating to the common QS-activated phenotypes, were screened from the 107 down-regulated genes (Fig. 4C). The result of quantitative PCR further confirmed that compared to the control, the expression levels of three key regulatory genes (IasR, $r / R$, and $p q s R$ ) and their downstream functional genes (las $B$, rhlA, pqsA, pqsD, pqsE, hcnA, and phzA) were all down-regulated by 1.5-8.2 folds in furazolidone-treated $P$. aeruginosa (Fig. 4D). Moreover, we also checked the effect of furazolidone on the expression levels of the main virulence factors of T. pyogenes TP13 and found that, the supplementation of furazolidone significantly decreased the expression of $p l o, p l o S, p l o R, c b p A$, fimA, nanH, and nanP by 1.7-10.7 folds in T. pyogenes TP13 compared to that of untreated group (Additional file 1 Supplementary Fig. S2).

\section{Furazolidone protects $\mathrm{C}$. elegans from $\mathrm{P}$. aeruginosa infection}

We then tested the in vivo protection activity of furazolidone against $P$. aeruginosa infection by using $C$. elegans as a model. In the fast-killing assay which mimics the acute infection condition, all the $C$. elegans were killed by $P$. aeruginosa PAO1 in 80 hours in the untreated group (Fig. 5A), while furazolidone treatment significantly increased the survival rate of $C$. elegans $(p<0.0001)$. In the slow-killing assay which mimics the chronic infection condition, $P$. aeruginosa PAO1 killed all the $C$. elegans in 8 days in the absence of furazolidone, while the death of $C$. elegans in furazolidone-treated group was slowed down in the initial 5 days and stopped on day 7 (Fig. 5B). Finally, the survival curve of $C$. elegans in furazolidonetreated group was significantly different from that of untreated group $(p=0.0102)$. Therefore, these results suggested that furazolidone could protect $C$. elegans from $P$. aeruginosa PAO1 challenge.

\section{Discussion}

Forest musk deer is a solitary ruminant vulnerable to various diseases and hurts from the environments, and among which the respiratory suppurative lesion is a major disease threatening the healthy growth of forest musk deer $[1,2]$. The present study identifies that furazolidone can simultaneously inhibit the growth and virulence of $T$. pyogenes and $P$. aeruginosa, which are the two important bacterial pathogens closely related to the occurrence and development of the respiratory suppurative disease of forest musk deer $[2,4,17]$.

Previous studies have reported that the QS signals of $P$. aeruginosa can inhibit the growth and virulence of $T$. pyogenes in vitro and in vivo [17, 21], and thus provides an explanation for the replacement of dominant bacteria from $T$. pyogenes to $P$. aeruginosa during the development of the respiratory 
suppurative disease of forest musk deer. This can also be supported by our current data that $P$. aeruginosa is ultimately the dominant strain in the mixed co-culture with $T$. pyogenes, irrespective of their initial ratios (Fig. 2). In comparison to the Gram-positive bacterium T. pyogenes which is normally isolated from animals and shows relatively less extent of antibiotic resistance, $P$. aeruginosa is a notorious Gramnegative bacterium with a variety of innate or acquired abilities to resist the clearance of antibiotics [22]. Moreover, reducing bacterial virulence by antagonizing QS system has been suggested to be an evolutionarily robust anti-infectious strategy $[16,23]$. These findings further hint that there might be a kind of drugs which is lethal to T. pyogenes but can inhibit the QS system of $P$. aeruginosa.

It is reported that the commonly used clinical antibiotics azithromycin, ceftazidime, and ciprofloxacin could function as QS inhibitors of $P$. aeruginosa to improve the clinical outcome of patients $[24,25,26]$. Some natural products such as the extract of Dalbergia Trichocarpa bark, baicalin (an active natural compound extracted from the traditional Chinese medicinal Scutellaria baicalensis), and sodium ascorbate could also inhibit the QS-regulated virulence of $P$. aeruginosa $[27,28,29]$. According to the principle deduced above, we first tested the antibacterial activities of 55 compounds with similar core structure to the AHL signals of $P$. aeruginosa QS system on mono-cultured T. pyogenes and co-cultured $T$. pyogenes and $P$. aeruginosa. Furazolidone, which could profoundly inhibit the growth of $T$. pyogenes TP13 and slightly inhibit $P$. aeruginosa PA01, was finally screened (Figs. 1 and 2). Furazolidone is a nitrofuran antibiotic that can be used to treat gastrointestinal diseases such as dysentery, enteritis, and gastric ulcer caused by bacteria and protozoa [30]. In the present study, we further discovered the antivirulence activity of furazolidone by showing that, the presence of furazolidone significantly reduced the production of QS-regulated virulence factors, biofilm formation, and cell motilities of $P$. aeruginosa PAO1 (Table 1 and Fig. 3). As determined by the transcriptomic analysis, furazolidone decreased the expression of 107 genes of $P$. aeruginosa PA01, and 63 of them were positively regulated by QS (Fig. 4A, C). The result of QPCR further demonstrated that furazolidone could inhibit the expression of all the three central regulatory genes (lasR, rh/R, and pqsR) and their downstream functional genes (lasB, rhIA, pqsA, pqsD, $p q s E$, hcnA, and phzA) of $P$. aeruginosa PAO1 (Fig. 4D). These data indicated that QS system was the major target inhibited by furazolidone in $P$. aeruginosa PA01.

On the other hand, the presence of furazolidone up-regulated the expression of 465 genes and significantly promoted the flagellar assembly, chemotaxis, and several metabolic pathways of $P$. aeruginosa PAO1 (Fig. 4B). Although it is not clear whether these enhanced abilities would contribute to the fitness of $P$. aeruginosa in the host tissue, the result of $C$. elegans killing experiments demonstrated that furazolidone was capable of efficiently protecting $C$. elegans from $P$. aeruginosa PAO1 infection, especially in the fast-killing assay (Fig. 5). We failed to measure the protection ability of furazolidone on $C$. elegans against $T$. pyogenes challenge. This is because the virulence of $T$. pyogenes is relatively moderate compared to the common bacterial pathogens and not sufficient to kill $C$. elegans even when the cell density was up to $1.0 \subseteq 10^{9} \mathrm{CFUs} / \mathrm{ml}$ (Data not shown). However, we found that furazolidone could inhibit the expression of the known virulence factor-encoding genes (Additional file 1 Supplementary Fig. S2). 


\section{Conclusion}

Collectively, our data here suggested that furazolidone could be considered as an antibacterial and antivirulence drug simultaneously inhibit the growth and virulence of $T$. pyogenes and $P$. aeruginosa. Further animal model-based mechanistic study combined with structure optimization of furazolidone would contribute to the development of novel drugs to treat the respiratory suppurative disease of forest musk deer.

\section{Methods}

\section{Bacterial strains and media}

T. pyogenes TP13 isolated from the lung pus of forest musk deer [4] and wild-type (WT) P. aeruginosa strain PA01 were preserved in the lab and used elsewhere [15]. All the strains were routinely cultured in brain heart infusion with $5 \%$ fetal bovine serum (BHI-FBS) or in lysogeny broth (LB) from a single colony.

\section{Culture conditions}

A total of 55 compounds (Additional file 1 Supplementary Table S1) with similar core structure to the Acyl-homoserine lactones (AHL) signals of $P$. aeruginosa QS system were selected and purchased from MedChemExpress (Shanghai, China). Overnight cultured T. pyogenes TP13 was diluted to optical density of 1.0 at wavelength of $600 \mathrm{~nm}\left(\mathrm{OD}_{600}=1.0\right)$ by sterile saline solution. Equal amount of $T$. pyogenes $(10 \mu \mathrm{L})$ was inoculated in $200 \mu \mathrm{L}$ of BHI-FBS medium containing different concentrations $(0$, 50,100 , and $200 \mu \mathrm{M}$ ) of compounds and culture at $37^{\circ} \mathrm{C}$ overnight, and then the cell densities were determined at $\mathrm{OD}_{600}$. Subsequently, T. pyogenes TP13 and $P$. aeruginosa PA01 were mixed $(1: 9,1: 1$, and 9:1) and co-cultured overnight on BHI-FBS agar containing $200 \mu \mathrm{M}$ of the compounds with significant growth inhibition activities on T. pyogenes. The composition of T. pyogenes and $P$. aeruginosa in the coculture were determined by counting the colony forming units (CFUs) of them on BHI-FBS agar plates after appropriate dilution, because the phenotypes of $T$. pyogenes and $P$. aeruginosa are significantly different and can be easily discriminated. Finally, the compounds that could inhibit the growth of $T$. pyogenes and $P$. aeruginosa were added $(0,50,100$, and $200 \mu \mathrm{M})$ to $200 \mu \mathrm{L}$ of LB medium containing 10 $\mu \mathrm{L}$ of $P$. aeruginosa and cultured overnight at $37^{\circ} \mathrm{C}$. The cell densities were determined at $\mathrm{OD}_{600}$. All the experiments above were independently repeated for three times.

\section{Quorum-sensing inhibition assay}

M9-adenisine (0.1\%, wt/v) and M9-skimmed milk (0.5\%, wt/v) agar medium were used to evaluate the inhibitory activity of compounds on $P$. aeruginosa QS regulation [19]. Overnight cultured $P$. aeruginosa PAO1 was adjusted to $\mathrm{OD}_{600}=1.0$ and inoculated $(5 \mu \mathrm{L})$ on $\mathrm{M} 9$-adenisine agar and M9-milk agar containing different concentrations $(0,50,100$, and $200 \mu \mathrm{M})$ of compounds. The growth status of $P$. aeruginosa on M9-adenisine plates and the diameter of proteolytic circle formed on M9-milk plates were determined after 24 hours. The experiments were independently repeated for nine times. 


\section{Biofilm production assay}

Equal amount $\left(20 \mu \mathrm{l}, \mathrm{OD}_{600}=1.0\right)$ of $P$. aeruginosa PAO1 was inoculated in glass tubes containing $2 \mathrm{~mL}$ of LB broth supplemented with different contractions $(0,50,100$, and $200 \mu \mathrm{M})$ of compounds, and overnight cultured at $37^{\circ} \mathrm{C}$ with shaking $(220 \mathrm{rpm})$. The cell density was measured at $\mathrm{OD}_{600}$. After the culture solution and unadhered biofilm were gently removed, the adhered biofilm on the tube wall was stained with crystal violet $(0.1 \%)$ for 30 minutes and washed with PBS buffer for three times. Subsequently, the stained biofilm was dissolved by $95 \%$ of ethanol solution and quantified at $\mathrm{OD}_{595}$. The experiments were independently repeated for three times.

\section{Pyocyanin production assay}

Equal amount $\left(20 \mu \mathrm{l}, \mathrm{OD}_{600}=1.0\right)$ of $P$. aeruginosa PAO1 was inoculated in glass tubes containing $2 \mathrm{~mL}$ of LB broth supplemented with different contractions $(0,50,100$, and $200 \mu \mathrm{M})$ of compounds, and overnight cultured at $37^{\circ} \mathrm{C}$ with shaking $(220 \mathrm{rpm})$. After the cell density was equalized with fresh LB broth, $200 \mu \mathrm{L}$ of bacterial solution was taken out to extract the pyocyanin by chloroform and $0.2 \mathrm{~N} \mathrm{HCl}$ and measured at $\mathrm{OD}_{520}$ as described by Essar [31]. The experiments were independently repeated for three times.

\section{Motility assay}

For the swimming motility assay, $5 \mu \mathrm{l}\left(\mathrm{OD}_{600}=1.0\right)$ of $P$. aeruginosa PAO1 was inoculated on the surface of LB plates containing $0.5 \%$ of agar supplemented with different contractions $(0,50,100$, and $200 \mu \mathrm{M})$ of compounds and cultured at $37^{\circ} \mathrm{C}$ for $24 \mathrm{~h}$. For the twitching motility assay, $2 \mu \mathrm{l}\left(\mathrm{OD}_{600}=1.0\right)$ of $P$. aeruginosa PAO1 was stabbed into the bottom of LB plates containing $1.0 \%$ of agar supplemented with different contractions $(0,50,100$, and $200 \mu \mathrm{M})$ of compounds and cultured at $37^{\circ} \mathrm{C}$ for $24 \mathrm{~h}$. The motilities of $P$. aeruginosa PAO1 were determined by measure the diameters of colony on the surface (swimming motility) or the thin film region on the bottom (twitching motility). All the experiments were independently repeated for six times.

\section{Transcriptomic analysis}

Bacterial cells of furazolidone-treated and -untreated $P$. aeruginosa PAO1 were harvested for total RNA isolation using TRIzol reagents (Invitrogen), respectively. RNAs samples were conducted for library construction and RNA-sequencing (RNA-seq) by Novogene Bioinformatics Technology Company using prokaryotic strand-specific Illumina-based RNA-Seq technology (HiseqTM2500 platform). The obtained clean reads were mapped to the reference genome of PA01 (NCBI accession number: AE004091) by the software Tophat2 [32]. SOAP2 program [33] and Cufflinks [34] were used to calculate the expected fragments per kilobase of transcript per million fragments (FPKM) sequenced, and the differentially expressed transcripts were presented and analyzed by EdgeR [35]. Differentially expressed gene with false discovery rate $p<0.05$ was thought to be significantly different. The significantly differently expressed genes were mapped to the list of QS-activated genes reported by Schuster [20] by using VENNY 2.1 (http:// bioinfogp.cnb.csic.es/tools/venny/). 


\section{Quantitative PCR}

Total RNAs of furazolidone-treated and -untreated $P$. aeruginosa PAO1 were isolated by using TRIzol reagents, and the CDNA was synthesized by reverse transcription using a high-capacity cDNA Reverse Transcriptase kit Specific with gDNA removal (Takara). Quantitative PCR was performed by using an $\mathrm{iTaq}^{\mathrm{TM}}$ universal SYBR ${ }^{\circledR}$ Green Supermix (Bio-Rad) and the CFX Connect Real-Time PCR Detection System to validate the expression of typical QS-activated genes including lasR, $r h / R, p q s R$, lasB, rhlA, pqsA, pqsD, $p q s E, h c n A$, and phzA (Additional file 1 Supplementary Table S2). Gene expression was calculated by the $2^{-\triangle \Delta C T}$ method using $16 \mathrm{~S}$ rRNA as reference.

\section{Caenorhabditis elegans assay}

For the fast-killing assay, $20 \mu \mathrm{l}\left(\mathrm{OD}_{600}=1.0\right)$ of $P$. aeruginosa PA01 was spread on peptone-glucosesorbitol (PGS) agar media with and without furazolidone, and cultured overnight at $37^{\circ} \mathrm{C}$. The naturally cooled plates were seeded with 10 newly cultured adult $C$. elegans ( $\mathrm{L} 4 \mathrm{stage}$ ) and further incubated at $25^{\circ} \mathrm{C}$ for 96 hours. For the slow-killing assay, to prevent $C$. elegans from laying eggs, $40 \mu \mathrm{L}$ of 5-fluoro-2'-

deoxyuridine solution $(40 \mu \mathrm{g} / \mathrm{mL})$ was evenly coated on the surface of nematode growth medium (NGM). Subsequently, $20 \mu \mathrm{l}\left(\mathrm{OD}_{600}=1.0\right)$ of $P$. aeruginosa PAO1 was spread on NGM plates with and without small molecule drugs and cultured overnight at $37^{\circ} \mathrm{C}$. The naturally cooled plates were seeded with 10 newly cultured adult $C$. elegans and further incubated at $25^{\circ} \mathrm{C}$ for 10 days. The survival status of $C$. elegans in each experiment were observed and recorded. Growth of $C$. elegans on PGS agar plates or NGM plates feed with uracil auxotrophy Escherichia coli OP50 were set as controls.

\section{Statistical analyses}

Data analysis and statistical tests were performed by using Graphpad Prism version 9.0 (San Diego, CA, USA). Mean values of standard deviation were compared by using two-tailed unpaired t-test or One-way ANOVA. The survival curves of $C$. elegans were compared by using Log- rank (Mantel-Cox) test.

\section{Abbreviations}

AHL: Acyl-homoserine lactones

BHI: brain heart infusion

CFUs: colony forming units

FBS: fetal bovine serum

FPKM: fragments per kilobase of transcript per million fragments

LB: lysogeny broth

NGM: nematode growth medium 
PCR: Polymerase chain reaction

PGS: peptone-glucose-sorbitol

QS: quorum-sensing

WT: wild-type

\section{Declarations}

\section{Ethics approval and consent to participate}

not applicable

\section{Consent for publication}

not applicable

\section{Availability of data and materials}

The data shown in this paper are available within the article and supplementary materials. The raw data of RNA- sequencing are available from the NCBI database under accession number PRJNA723215 (SRR14368535 and SRR14368537) https://www.ncbi.nlm.nih.gov/sra/?term=PRJNA723215.

\section{Competing interests}

The authors declare no conflict of interest exists.

\section{Funding}

This work was supported by the National Natural Science Foundation of China (31970131), the Sichuan Association for Science and Technology (2018RCTJ), the Sichuan Science and Technology Program (2018HH0007).

\section{Authors' contributions}

Experiment and data processing: Q. CHEN. K. L. ZHAO. H. Y. LI. K. H. LIU. J. LI. Wrote the manuscript: Q. CHEN. Revised the manuscript: K. L. ZHAO. Y.W.CHU. B. Prithiviraj. B. S. YUE. X. Y. ZHANG. The author(s) read and approved the final manuscript.

\section{Authors' information}

Key Laboratory of Bio-resources and Eco-environment, Ministry of Education, College of Life Sciences, Sichuan University, Chengdu, 610064, China

Qin Chen, Heyue Li, Kanghua Liu, Bisong Yue, Xiuyue Zhang 
Antibiotics Research and Reevaluation Key Laboratory of Sichuan Province, College of Pharmacy, Sichuan Industrial Institute of Antibiotics, Chengdu University, Chengdu 610052, China

Kelei Zhao, Jing Li, Yiwen Chu

Marine Bio-products Research Laboratory, Department of Plant, Food and Environmental Sciences, Dalhousie University, Truro, NS, Canada

Balakrishnan Prithiviraj

Acknowledgements

not applicable

\section{References}

1. Zhao KL, Li XX, Palahati P, Zhang XY, Zeng B, Yue BS. Isolation and identification on pathogens of musk-deer abscess disease and antibiotic susceptibility assay. Sichuan. J. Zool. 2011a,30:522-6 in Chinese, with English abstract.

2. Zhao KL, Liu Y, Zhang XY, Palahati P, Wang HN, Yue BS. Detection and characterization of antibiotic resistance genes in Arcanobacterium pyogenes strains from abscesses of forest musk deer. J. Med. Microbiol. 2011b,60:1820-6.

3. Zhao KL, Ma JN, Wang XY, Guo YD, Yue BS, Chu YW. Population divergence of Pseudomonas aeruginosa can lead to the coexistence with Escherichia coli in animal suppurative lesions. Vet. Microbiol. 2019,231:169-76.

4. Zhao KL, Liu MY, Zhang XY, Wang HN, Yue BS. In vitro and in vivo expression of virulence genes in Trueperella pyogenes based on a mouse model. Vet. Microbiol. 2013,163:344-50.

5. Jost BH, Billington SJ. Arcanobacterium pyogenes: Molecular pathogenesis of an animal opportunist. Antonie. Van. Leeuwenhoek. 2005, 88:87-102.

6. Zhao KL, Li WJ, Kang CL, Du LM, Huang T, Zhang XY, et al. Phylogenomics and evolutionary dynamics of the family Actinomycetaceae. Genome. Biol. Evol. 2014,6:2625-33.

7. Belser EH, Cohen BS, Keeler SP, Killmaster CH, Bowers JW, Miller KV. Epethelial presence of Trueperella pyogenes predicts site-level presence of cranial abscess disease in white-tailed deer (Odocoileus virginianus). PLoS ONE. 2015,10:e0120028.

8. Rzewuska M, Kwiecień E, Chrobak-Chmiel D, Kizerwetter-Świda M, Stefańska I, Gieryńska M. Pathogenicity and Virulence of Trueperella pyogenes: A Review. Int. J. Mol. Sci. 2019,20:2737.

9. Roberts DS. The pathogenic synergy of Fusiformis necrophorus and Corynebacterium pyogenes. II. The response of F. necrophorus to a filterable product of $C$. pyogenes. Br. J. Exp. Pathol. 1967,48: 674-9. 
10. Bicalho ML, Machado VS, Oikonomou G, Gilbert RO, Bicalho RC. Association between virulence factors of Escherichia coli, Fusobacterium necrophorum, and Arcanobacterium pyogenes and uterine diseases of dairy cows. Vet. Microbiol. 2012,157:125-31.

11. Moradali MF, Ghods S, Rehm BH. Pseudomonas aeruginosa lifestyle: a paradigm for adaptation, survival, and persistence. Front. Cell. Infect. Microbiol. 2017,7:39.

12. Valentini M, Gonzalez D, Mavridou DA, Filloux A. Lifestyle transitions and adaptive pathogenesis of Pseudomonas aeruginosa. Curr. Opin. Microbiol. 2017,41:15-20.

13. Rutherford ST, Bassler BL. Bacterial quorum sensing: Its role in virulence and possibilities for its control. Cold. Spring. Harb. Perspect. Med. 2012,2:a012427.

14. Balasubramanian $D$, Schneper $L$, Kumari $H$, Mathee $K$. A dynamic and intricate regulatory network determines Pseudomonas aeruginosa virulence. Nucleic. Acids. Res. 2013,41:1-20.

15. Zhao KL, Du LM, Lin JF, Yuan Y, Wang XY, Yue BS, et al. Pseudomonas aeruginosa quorum-sensing and type VI secretion system can direct interspecific coexistence during evolution. Front. Microbiol. 2018,9:2287.

16. Defoirdt T. Quorum-sensing systems as targets for antivirulence therapy. Trends Microbiol. 2018,26:313-28.

17. Zhao KL, Li WJ, Huang T, Song $X H$, Zhang $X Y$, Yue BS. Comparative transcriptome analysis of Trueperella pyogenes reveals a novel antimicrobial strategy. Arch. Microbiol. 2017,199:649-55.

18. Yuan Y, Li J, Zhang AX, Lin JF, Chu YW, Wang XY, et al. Interspecific Interaction Between Pseudomonas aeruginosa and Trueperella pyogenes From the Abscesses Disease of Moschus berezovskii. Sichuan. J. Zool. 2020,39:241-8 in Chinese, with English abstract.

19. Darch SE, West SA, Winzer K, Diggle SP. Density-dependent fitness benefits in quorum-sensing bacterial populations. Proc. Natl. Acad. Sci. U. S. A. 2012,109:8259-63.

20. Schuster M, Lostroh CP, Ogi T, Greenberg EP. Identification, timing, and signal specificity of Pseudomonas aeruginosa quorum-controlled genes: a transcriptome analysis. J. Bacteriol. 2003,185:2066-79.

21. Huang T, Song $X H$, Zhao KL, Jing J, Shen YM, Zhang XY, et al. Quorum-sensing molecules $\mathrm{N}$-acyl homoserine lactones inhibit Trueperella pyogenes infection in mouse model. Vet. Microbiol. 2018,213:89-94.

22. Azam MW, Khan AU. Updates on the pathogenicity status of Pseudomonas aeruginosa. Drug. Discov. Today. 2019,24:350-9.

23. Allen RC, Popat R, Diggle SP, Brown SP. Targeting virulence: can we make evolution-proof drugs? Nat. Rev. Microbiol. 2014,12:300-8.

24. Skindersoe ME, Alhede M, Phipps R, Yang L, Jensen PO, Rasmussen TB, et al. Effects of antibiotics on quorum sensing in Pseudomonas aeruginosa. Antimicrob. Agents. Chemother. 2008,52:3648-63.

25. Otani S, Hiramatsu K, Hashinaga K, Komiya K, Umeki K, Kishi K, et al. Sub-minimum inhibitory concentrations of ceftazidime inhibit Pseudomonas aeruginosa biofilm formation. J. Infect. 
Chemother. 2018,24:428-33.

26. Rehman A, Patrick WM, Lamont IL. Mechanisms of ciprofloxacin resistance in Pseudomonas aeruginosa: new approaches to an old problem. J. Med. Microbiol. 2019,68:1-10.

27. Rasamiravaka T, Jedrzejowski A, Kiendrebeogo M, Rajaonson S, Randriamampionona D, Rabemanantsoa C, et al. Endemic malagasy Dalbergia species inhibit quorum sensing in Pseudomonas aeruginosa PA01. Microbiology. 2013,159(Pt 5):924-38.

28. El-Mowafy SA, Shaaban MI, Abd El Galil KH. Sodium ascorbate as a quorum sensing inhibitor of Pseudomonas aeruginosa. J. Appl. Microbiol. 2014,117:1388-99.

29. Luo J, Dong BY, Wang K, Cai SQ, Liu TJ, Cheng XJ, et al. Baicalin inhibits biofilm formation, attenuates the quorum sensing-controlled virulence and enhances Pseudomonas aeruginosa clearance in a mouse peritoneal implant infection model. PLoS One. 2017,12:e0176883.

30. Altamirano A, Bondani A. Adverse reactions to furazolidone and other drugs. A comparative review. Scand. J. Gastroenterol Suppl. 1989,169:70-80.

31. Essar DW, Eberly L, Hadero A, Crawford IP. Identification and characterization of genes for a second anthranilate synthase in Pseudomonas aeruginosa: interchangeability of the two anthranilate synthases and evolutionary implications. J. Bacteriol. 1990,172:884-900.

32. Kim D, Pertea G, Trapnell C, Pimentel H, Kelley R, Salzberg SL. TopHat2: accurate alignment of transcriptomes in the presence of insertions, deletions and gene fusions. Genome. Biol. 2013,14: R36.

33. Li RQ, Yu C, Li YR, Lam TW, Yiu SM, Kristiansen K, et al. SOAP2: an improved ultrafast tool for short read alignment. Bioinformatics. 2009,25:1966-7.

34. Mortazavi A, Williams BA, McCue K, Schaeffer L, Wold B. Mapping and quantifying mammalian transcriptomes by RNA-Seq. Nat. Methods. 2008,5:621-28.

35. Robinson MD, McCarthy DJ, Smyth GK. EdgeR: a Bioconductor package for differential expression analysis of digital gene expression data. Bioinformatics. 2009,26:139-140.

\section{Figures}


A

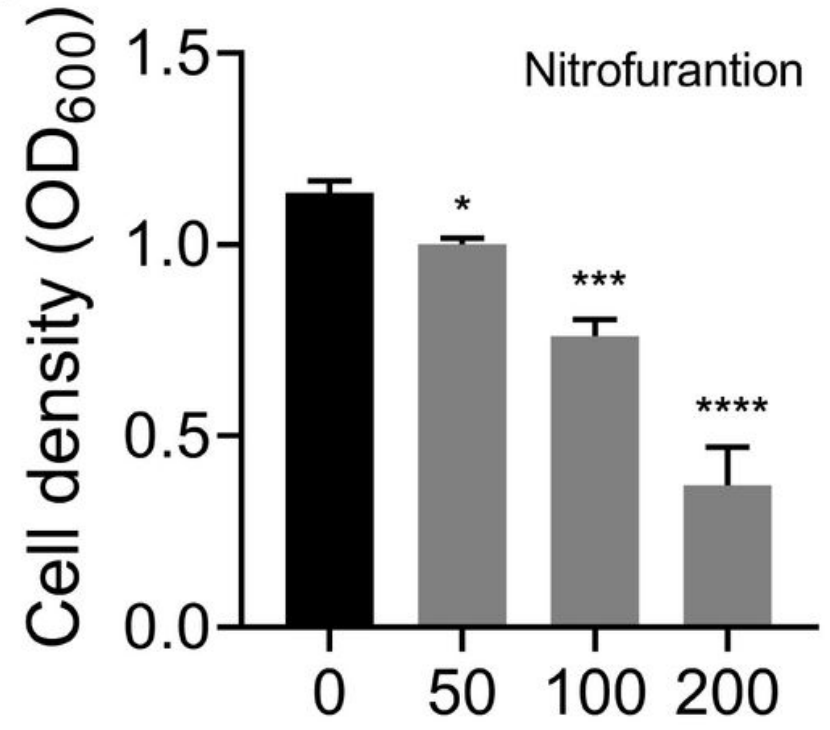

$\mu \mathrm{M}$

C

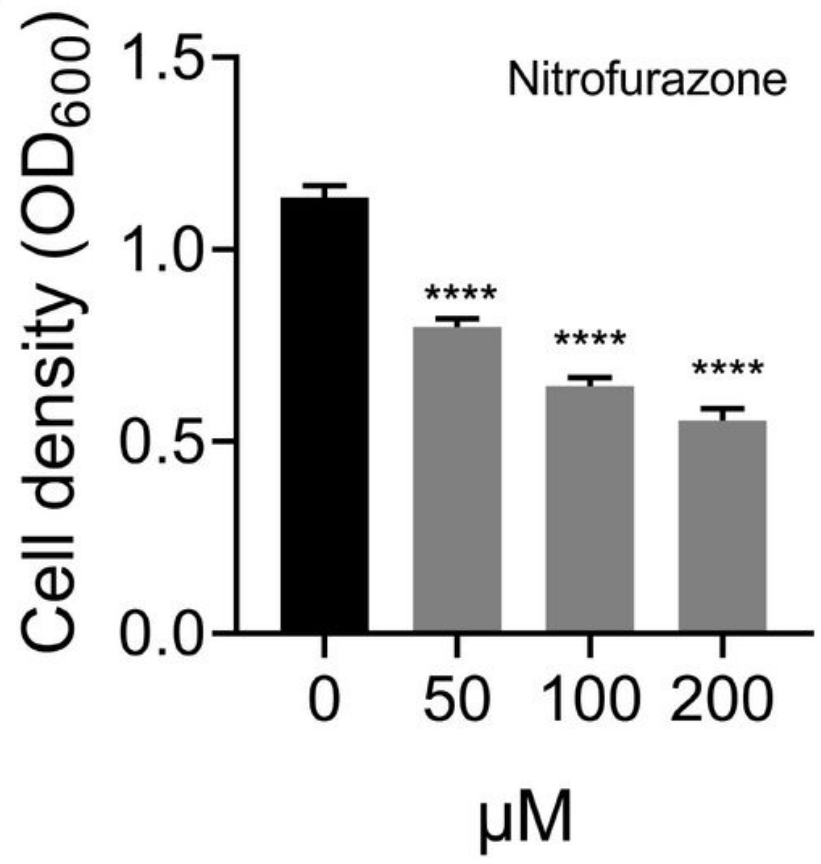

$\mathrm{B}$

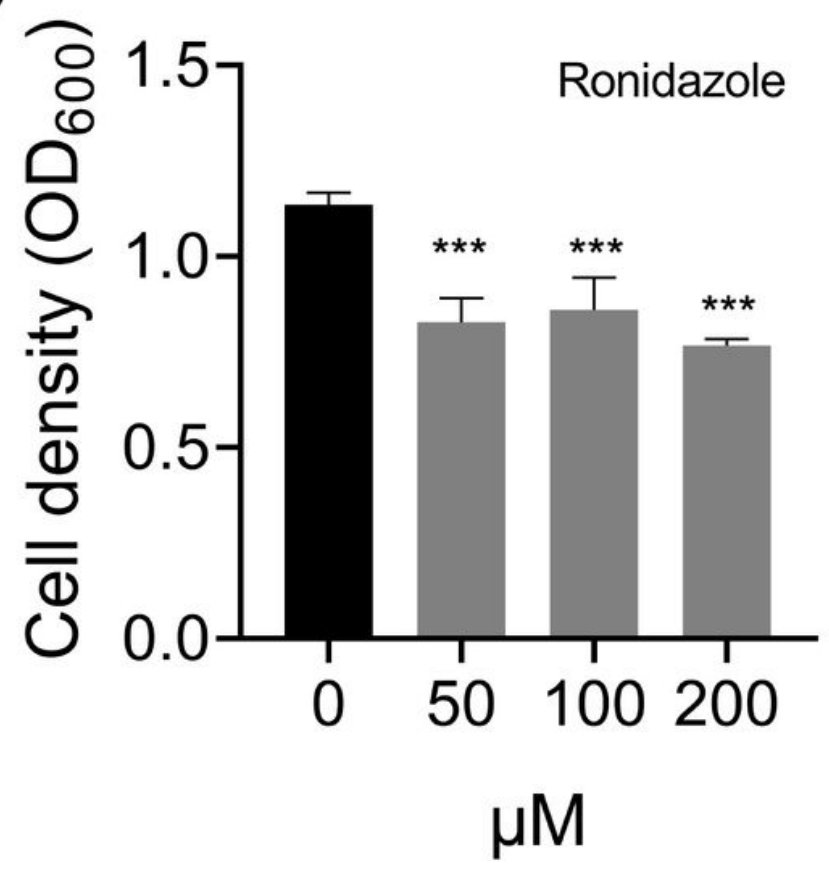

D

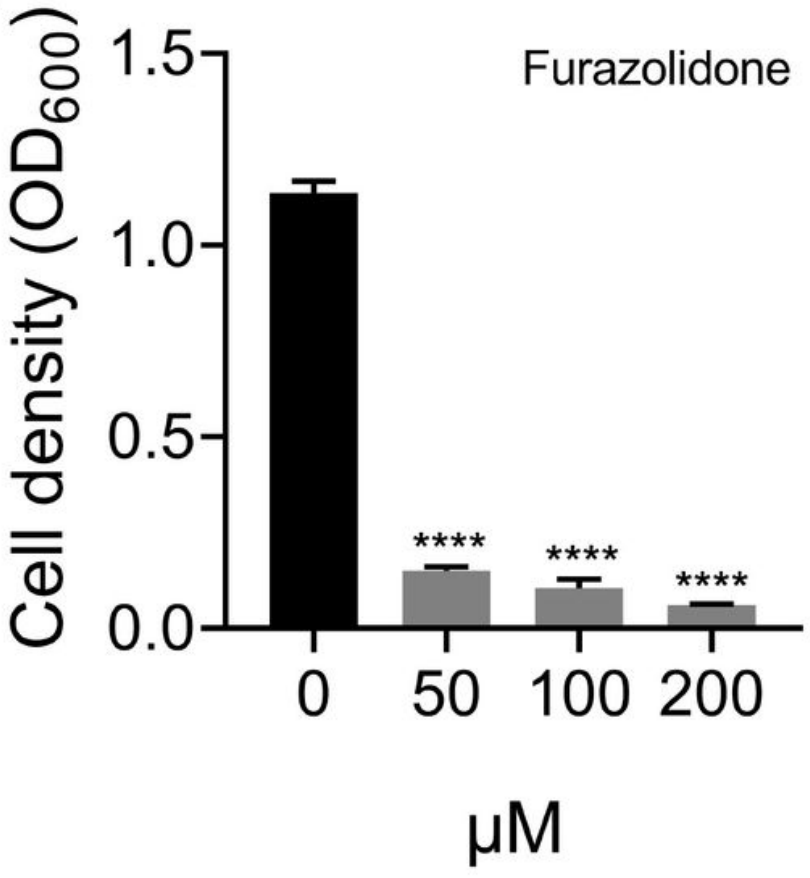

Figure 1

Effects of different compounds on the growth of Trueperella pyogenes TP13. Equal amount of T. pyogenes TP13 was inoculated in brain heart infusion $(\mathrm{BHI})$ broth containing $5 \%$ fetal bovine serum (FBS) and different concentrations of (A) nitrofurantoin, (B) ronidazole, (C) nitrofurazone, or (D) furazolidone, and cultured overnight at $37^{\circ} \mathrm{C}$. Data shown are the mean \pm standard deviation (SD) of three independent experiments. One-way ANOVA, ${ }^{*} p<0.05,{ }^{* * *} p<0.001$, ${ }^{\star * \star *} p<0.0001$. 

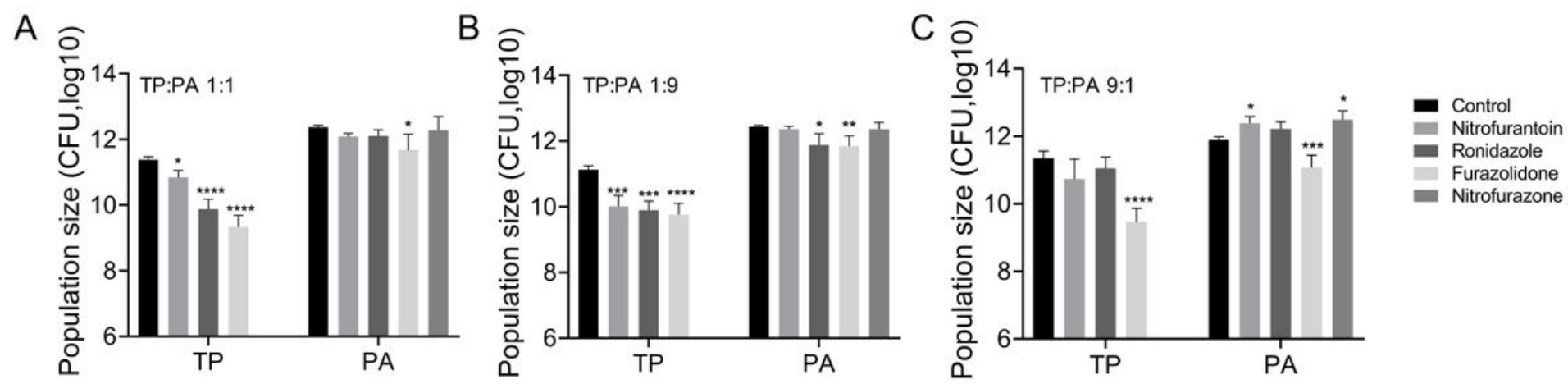

Figure 2

Effects of different compounds on the growth of T. pyogenes TP13 and Pseudomonas aeruginosa PA01 under different co-culture conditions. T. pyogenes TP13 and P. aeruginosa PAO1 were mixed and cocultured on $\mathrm{BH}+5 \% \mathrm{FBS}$ plates containing $200 \mu \mathrm{M}$ of nitrofurantoin, ronidazole, nitrofurazone, or furazolidone from the initial ratios of (A) 1:1, (B) 1:9, and (C) 9:1, and cultured overnight at $37^{\circ} \mathrm{C}$. Data shown are the mean $\pm S D$ of three independent experiments. One-way ANOVA, ${ }^{*} p<0.05, * \star p<0.01, * \star * p$ $<0.001, * \star \star \star p<0.0001$. 
A

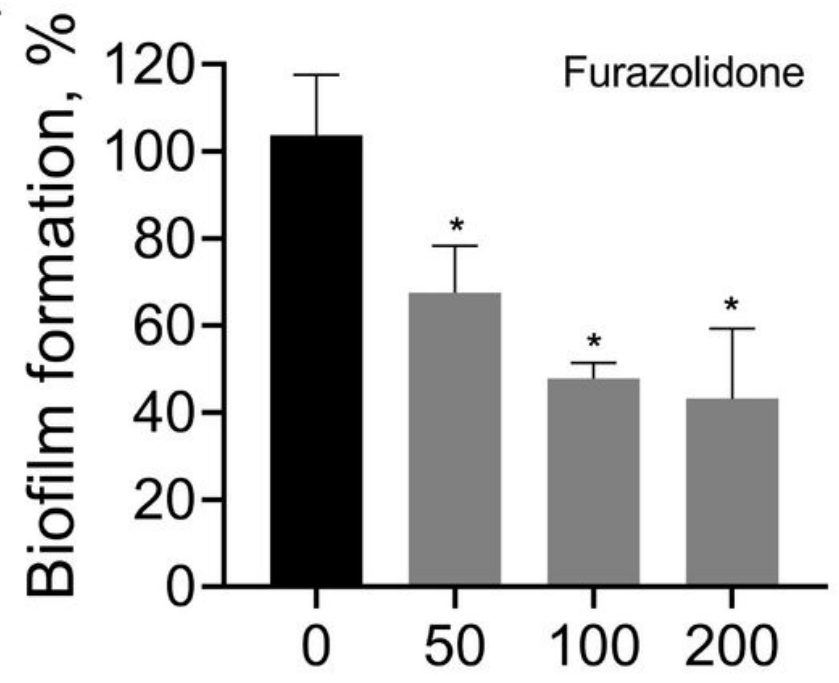

$\mu \mathrm{M}$

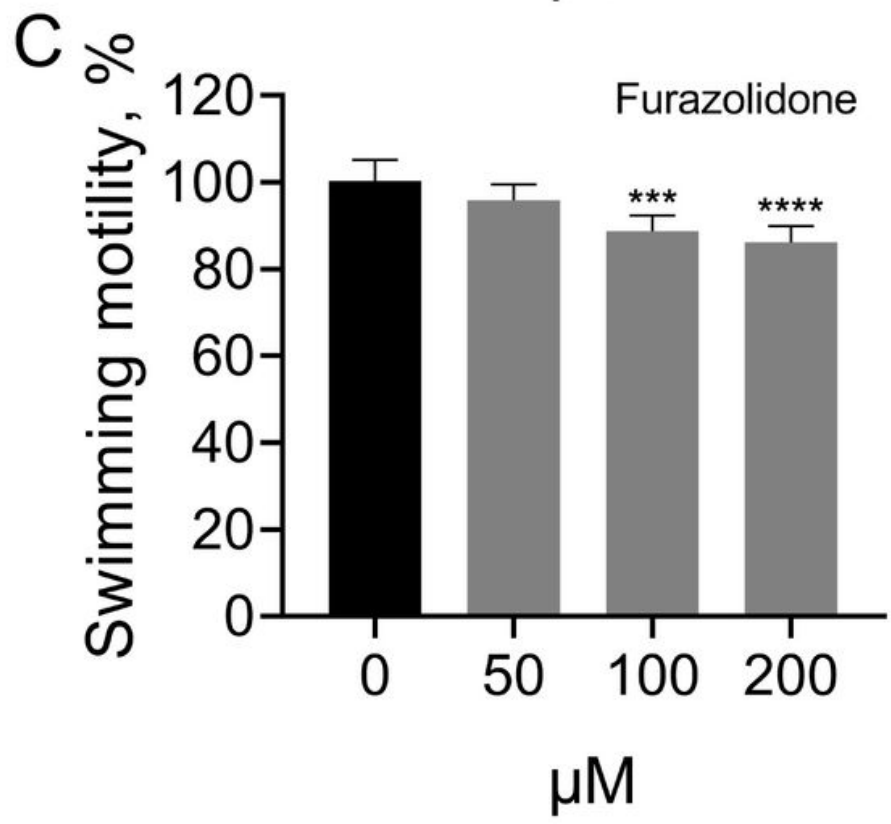

$\mathrm{B} \curvearrowright 0$

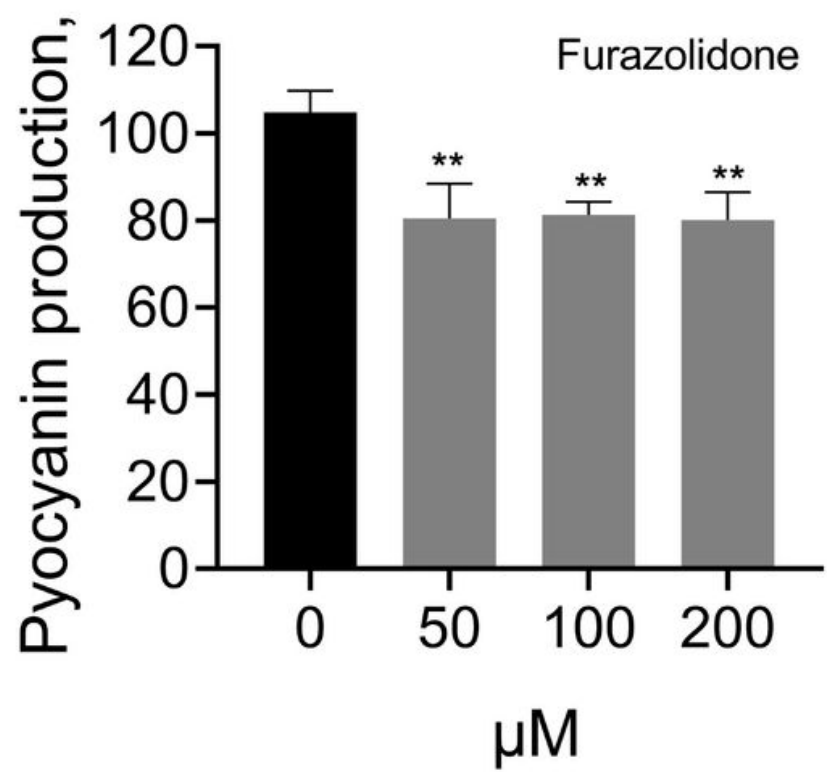

$\mathrm{D}$

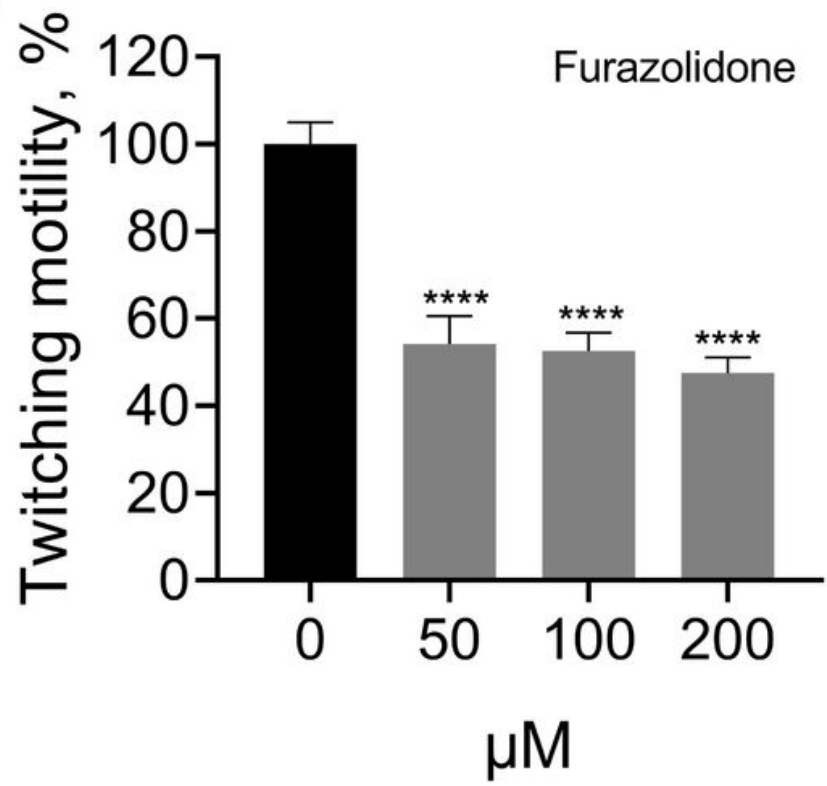

Figure 3

Inhibitory effect of furazolidone on the (A) biofilm formation, (B) pyocyanin production, (C) swimming motility and (D) twitching motility of P. aeruginosa PA01. Data shown are the mean $\pm S D$ of three independent experiments. One-way ANOVA, ${ }^{\star} p<0.05$, ${ }^{\star \star} p<0.01$, ${ }^{\star \star \star} p<0.001$, ${ }^{\star \star \star \star} p<0.0001$. 
A

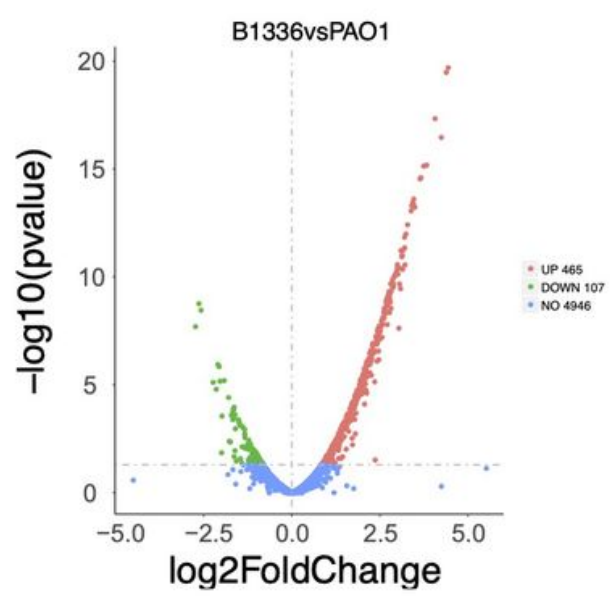

C

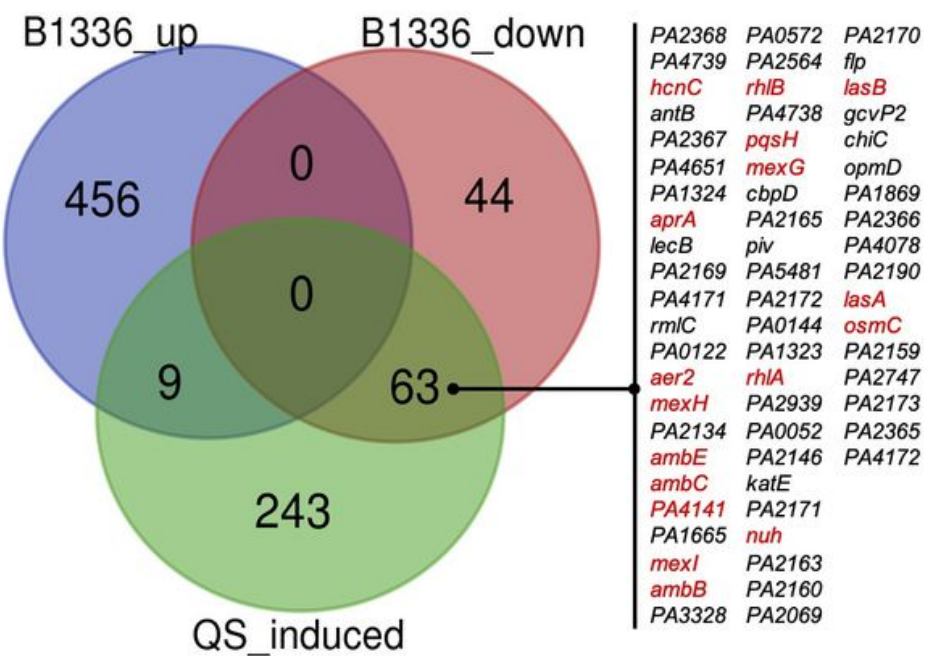

B

\begin{tabular}{|l|c|}
\hline KEGG Pathway & $\begin{array}{c}p \text {-value } \\
\text { (Corrected) }\end{array}$ \\
\hline Up-regulated (gene No.) & \\
Flagellar assembly (14) & 0.0002 \\
Bacterial chemotaxis (13) & 0.0006 \\
Propanoate metabolism (11) & 0.009 \\
Ribosome (12) & 0.011 \\
Valine, leucine and isoleucine & 0.037 \\
Degradation (10) & \\
\hline Down-regulated & 0.00008 \\
Quorum sensing (10) & \\
\hline
\end{tabular}

D

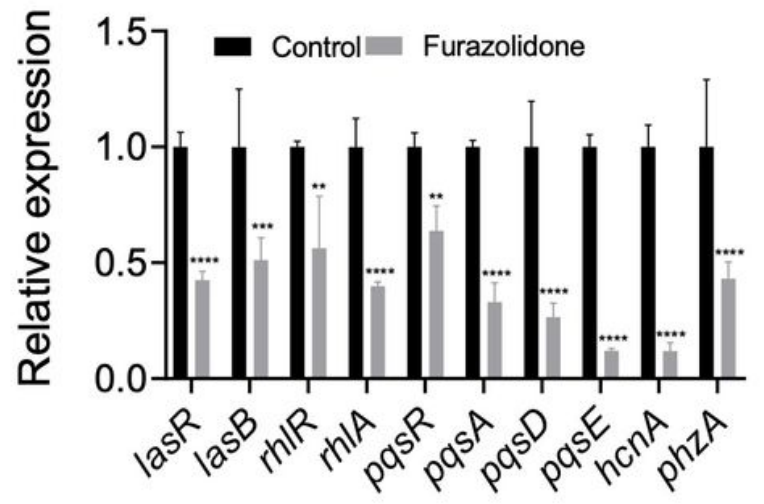

\section{Figure 4}

Effects of furazolidone on the global transcription of P. aeruginosa PA01. (A) Volcano plotting of differentially expressed genes. B1336 indicates furazolidone treated PA01. (B) Significantly influenced KEGG pathways of P. aeruginosa PAO1 by furazolidone. (C) The significantly differentially expressed genes of P. aeruginosa PA01 were applied to the list of QS-induced genes published by Schuster et al. (2003). (D) Expression of typical QS-regulated genes of furazolidone-treated PAO1 as determined by qPCR. Data shown are the mean \pm SD of three independent experiments. One-way ANOVA, $* \star p<0.01, * \star *$ $p<0.001, * \star \star * p<0.0001$.
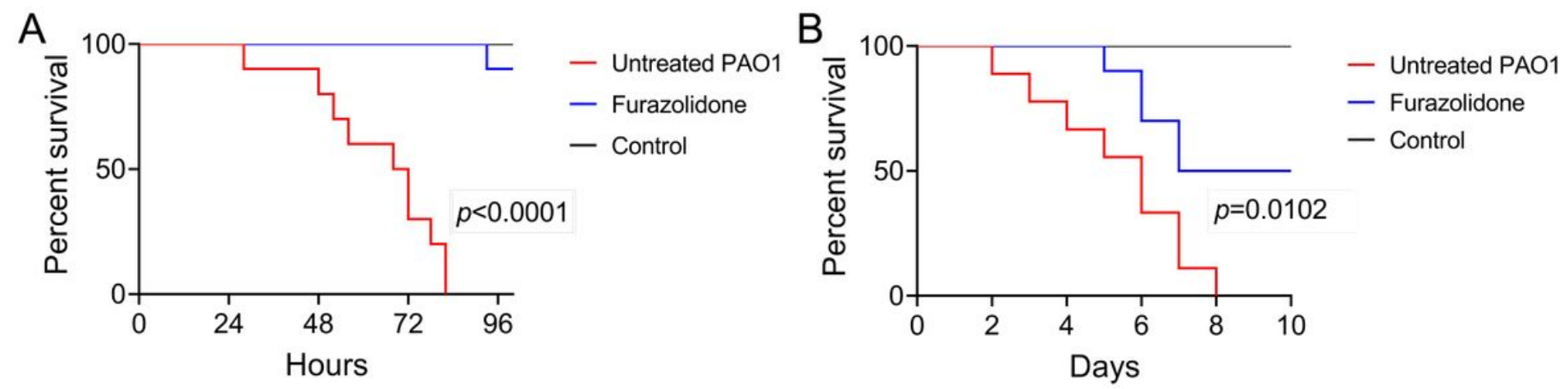
Figure 5

Pathogenicity of P. aeruginosa PAO1 can be reduced by furazolidone. Survival of Caenorhabditis elegans models challenged by P. aeruginosa PAO1 under (A) fast-killing and (B) slow-killing conditions (10 nematodes per group). Data shown are representative of six independent replicates. The survival curves were compared by using Log-rank (Mantel-Cox) test.

\section{Supplementary Files}

This is a list of supplementary files associated with this preprint. Click to download.

- Additionalfile1.docx

- Additionalfile2.xlsx 